\title{
Keeping an open mind: highlights and controversies of the breast cancer stem cell theory
}

\author{
Mansi Shah' \\ Cinzia Allegrucci ${ }^{1,2}$ \\ 'School of Veterinary Medicine and \\ Science, University of Nottingham, \\ Sutton Bonington Campus, \\ Loughborough, UK; ${ }^{2}$ Center for \\ Genetics and Genomics and Cancer \\ Research Nottingham, University \\ of Nottingham, University Park, \\ Nottingham, UK
}

This article was published in the following Dove Press journal:

Breast Cancer:Targets and Therapy

25 October 2012

Number of times this article has been viewed

\begin{abstract}
The discovery that breast cancers contain stem-like cells has fuelled exciting research in the last few years. These cells are referred to as breast cancer stem cells (BCSCs) and are thought to be involved in tumor initiation, progression, and metastasis. Being intrinsically resistant to chemo- and radiotherapy, they are also considered responsible for recurrence of the disease after treatment. BCSCs have been suggested to be at the basis of tumor complexity, as they have the ability to self-renew and give rise to highly proliferating and terminally differentiated cancer cells that comprise the heterogeneous bulk of the tumor. There has been much speculation on the BCSC model, and in this review we address some fundamental questions, such as the identity of BCSCs and their involvement in tumor intra- and interheterogeneity. As an alternative to the BCSC model, we discuss clonal evolution, as both theories show extensive evidence in support of their arguments. Finally, we discuss a unifying idea that reconciles both models, which is based on stem cell plasticity and epigenetic modifications induced by the tumor microenvironment. The implications of cancer stem cell plasticity for drug discovery and future therapeutic interventions are presented.
\end{abstract}

Keywords: mammary stem cells, cancer, clonal evolution, plasticity, therapy, epigenetics

\section{Breast cancer: a complex and heterogeneous disease}

Breast cancer is one of the most common causes of cancer-related mortality in women worldwide (following lung cancer), with more than one million women diagnosed every year, and half a million dying from this disease. Although medical advances have contributed to early detection and better treatment, the mortality rate of women with breast cancer is still relatively high due to recurrence and metastasis. ${ }^{1}$ Breast cancer represents a major clinical challenge as it is a complex disease, and presents with significant variability in tissue histopathology, metastatic behavior, response to treatment, and patient outcomes. At a cellular level, breast cancer is regarded as a heterogeneous disease. This heterogeneity profoundly impacts treatment, as combinatorial therapies are required to target different cancer cells. Heterogeneity is not only a feature of different breast tumor subtypes (interheterogeneity) but also of the same tumor (intraheterogeneity). ${ }^{2}$ In terms of interheterogeneity, different breast cancer subtypes can be classified based on clinical and histological factors, which include tumor grade, size, stage, and lymph node metastasis. According to the World Health Organization, there are at least 18 different histological subtypes of breast cancer. ${ }^{3}$ Although these histological and clinical subsets give a detailed account of different tumors, there is still variability with grading and diagnosis.
Correspondence: Cinzia Allegrucci

University of Nottingham, SVMS,

Sutton Bonington Campus,

Loughborough, LEI 2 5RD, UK

Tel +44 II 595 I 6487

Fax +44 II 595। 6440

Email cinzia.allegrucci@nottingham.ac.uk 
Molecular profiling of tumors shows that breast cancer is a heterogeneous set of different diseases, determined by various molecular alterations, rather than being a single disease with multiple manifestations. ${ }^{4}$ Genetic and epigenetic insults contribute to breast carcinogenesis. ${ }^{5}$ These alterations cause aberrant expression of oncogenes and silencing of tumor suppressor genes with consequent disruption in gene networks regulating normal tissue homeostasis, such as cell proliferation, differentiation, motility, apoptosis, and growth. ${ }^{6}$ Gene expression profiling performed across breast cancer subsets identified estrogen receptor alpha (ER) positive $\left(\mathrm{ER}^{+}\right)$and negative $\left(\mathrm{ER}^{-}\right)$tumors as two distinct cancer types. Furthermore, it allowed further subclassification across five molecular subtypes depending on their "intrinsic gene expression" signature. These include normal-like, luminal A, luminal B, HER2 positive (HER2 $2^{+}$), and basal-like subtypes. ${ }^{7-9}$ Luminal A tumors are defined by the expression of both $\mathrm{ER}^{+}$and/or progesterone receptors $\left(\mathrm{PR}^{+} / \mathrm{PR}^{-}\right)$, and by the absence of HER2 amplification. Luminal B tumors are similar to luminal A, but include the amplification of HER2. HER $2^{+}$tumors are defined by HER 2 expression and may lack the expression of ER and PR. Basal-like tumors are defined by the absence of ER, PR, and HER2 expressions and are further subdivided into basal A and basal B. ${ }^{7,10}$ More recently, a molecular signature identified as 'claudin-low' has been found to overlap with the basal B subtype. Claudin-low tumors lack the expression of ER, PR, and HER2, and are therefore also identified as "triple negative." Normal-like breast cancer shows a gene signature similar to that of normal breast tissue. ${ }^{11}$ Less common than other subtypes, normallike cancers are of an ambiguous origin, and it is still debated whether they may represent breast tissue containing too few detectable cancer cells at the time of analysis. ${ }^{12}$

Breast cancer subtypes exhibit differences in the incidence of the disease, survival rates, and response to treatment. Luminal tumors (almost exclusively $\mathrm{ER}^{+}$) are the most common, and are associated with positive outcomes as they are treatable with hormonal therapy (tamoxifen). Luminal A subtypes are less proliferative than luminal B, and therefore have a better outcome. HER $2^{+}$tumors are highly proliferative, and present with worse outcomes even if treated with the

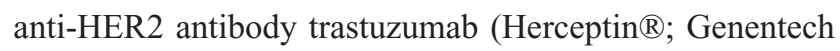
USA, Inc, San Francisco, CA). Basal-like breast cancers represent high grade cancers with poor patient outcomes. Although somewhat sensitive to chemotherapy, these cancers are associated with high levels of recurrence after treatment. Targeted therapies, with the exception of poly (ADP-ribose) polymerase (PARP) inhibitors, are lacking for basal-like breast cancers (Table 1). ${ }^{13}$ New research has revealed that the complexity of breast cancer is higher than previously expected. Indeed, a screening of 2000 breast tumors that combined inherited and acquired genetic alterations with gene expression data, highlighted a novel molecular stratification of tumors with ten different subtypes. ${ }^{14}$

The cellular origin of different breast tumor subtypes is still unclear. Some breast cancer subtypes have similar genetic and molecular compositions as their normal mammary cell counterparts. For instance, luminal subtypes have a similar molecular makeup to luminal mature nonclonogenic cells in that they are both $\mathrm{ER}^{+}$and $\mathrm{PR}^{+}$. They also express characteristic luminal markers, such as CK18, CK19, CD24, MUC1, and ESA. In contrast, the basal B/ claudin-low subtypes lack the ER, PR, and HER2 expression and express markers characteristic of the basal lineage, such as CK14, CD49f, and CD44. ${ }^{15}$ This evidence would suggest that different cancer subtypes originate from either luminal or basal/myoepithelial progenitors within the normal tissue. However, this correlation does not exist when basal-like/BRCA1 mutant tumors are considered, as they seem to originate from luminal progenitor cells, rather than from basal progenitors. ${ }^{16,17}$ The origin of all tumor subtypes - whether it is a cell with multilineage potential,

Table I Biological and clinical characteristics of breast cancer molecular subtypes

\begin{tabular}{|c|c|c|c|c|c|c|}
\hline Subtype & Markers & Prevalence & CSC enrichment & Targeted treatment & Mechanism & Prognosis \\
\hline Luminal $\mathrm{A}$ & $\begin{array}{l}\text { Mostly ER }{ }^{+} \text {and/or } \mathrm{PR}^{+} \\
\left(\text {some HER2 } 2^{+}\right)\end{array}$ & $42 \%-59 \%$ & Low & Tamoxifen & ER & Good \\
\hline Luminal B & $\begin{array}{l}\text { Mostly } \mathrm{ER}^{+} \text {and/or } \mathrm{PR}^{+} \\
\text {(some } \mathrm{HER2} 2^{+} \text {) } \\
\text { Highly proliferative }\end{array}$ & $6 \%-19 \%$ & Intermediate & $\begin{array}{l}\text { Tamoxifen, } \\
\text { Herceptin }\end{array}$ & $\begin{array}{l}\text { ER, } \\
\text { HER2 }\end{array}$ & Intermediate \\
\hline HER2 $^{+}$ & $\begin{array}{l}\mathrm{HER}^{+} \\
\text {(can be } \mathrm{ER}^{+} / \mathrm{ER}^{-} \text {and } \mathrm{PR}^{+} / \mathrm{PR}^{-} \text {) }\end{array}$ & $7 \%-12 \%$ & High & $\begin{array}{l}\text { Lapatinib, } \\
\text { Herceptin }\end{array}$ & HER2 & Poor \\
\hline $\begin{array}{l}\text { Basal-like } \\
\text { triple negative }\end{array}$ & $\begin{array}{l}\text { Mostly ER-/PR-/HER2 } \\
\mathrm{CK}^{+} / 6^{+} \text {, and/or HER I+ }\end{array}$ & $14 \%-20 \%$ & High & $\begin{array}{l}\text { Bevacizumab, } \\
\text { PARP inhibitors }\end{array}$ & HER 1 & Poor \\
\hline
\end{tabular}

Abbreviations: ER, estrogen receptor alpha; PR, progesterone receptor; HER2, epidermal growth factor receptor 2; HERI, epidermal growth factor receptor I. 
a progenitor cell, or even a differentiated cell - is still under debate. Moreover, this quest is further complicated by the occurrence of intratumor heterogeneity within breast cancer subtypes. Intraheterogeneity is typically observed at the histological level, with different cell types and/or morphological appearances making up the bulk of the tumor. This is reflected in variable gene expression signatures and is best exemplified by the variable ER expression within a patient tumor. ${ }^{18}$ Two different models, supported by experimental findings, explain the origin of tumor heterogeneity: stem cell hierarchy and clonal evolution.

\section{Breast cancer stem cells}

The idea that cancer is driven by cells with stem cell-like features is not new, but has received renewed interest in recent years. The theory that cancer arises from stem cells was developed in the late nineteenth century when a correlation between embryonic stem cells and cancer was established among teratocarcinomas. ${ }^{19}$ The existence of malignant stem cells, also known as cancer stem cells (CSCs), was first discovered in acute myeloid leukemia; since then, CSCs have been identified and isolated in many solid tumors including breast, prostate, brain, and lung. ${ }^{20}$ According to the CSC hypothesis, the tumor is organized into aberrant hierarchies in which the CSC lies at the apex, and highly proliferating progenitors and terminally differentiated cancer cells reside at the bottom. In this model, CSCs would sustain tumor growth by symmetrical and asymmetrical self-renewal, whereas lineage-committed progenitor and differentiated cells would make up the heterogeneous bulk of the tumor (Figure1).

The CSC hierarchy model predicts that CSCs originate from the transformation of normal stem cells and, in this context, they are believed to be a rare population of cells more tumorigenic than the non-CSC population. ${ }^{20}$ However, the origin of CSC is at the center of controversy, as they may not necessarily derive from transformed stem cells, as the name implies. Therefore, many researchers prefer to refer to these cells as 'cancer-initiating cells' or 'cancer-propagating cells.' For the simplicity of semantics, we shall refer to them as CSCs in this review. ${ }^{21}$ The cell-of-origin of breast CSCs (BCSCs) is not yet known, but there are two possibilities: they either originate from undifferentiated mammary stem cells (MaSCs) or committed stem/progenitor cells through genetic and epigenetic reprogramming. ${ }^{21}$

The mammary gland is a very dynamic tissue and it is organized in a hierarchical fashion where MaSCs give rise to highly proliferating progenitors and differentiated cells of the epithelial and myoepithelial lineages (Figure 2). Most of our knowledge about MaSCs comes from the mouse model, where experiments of serial transplantation into the mammary fat pad show that single basal stem cells, identified

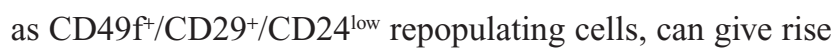
to different mammary structural units. ${ }^{22,23}$ According to the BCSC theory, it is thought that long-living MaSCs might represent a likely target for malignant transformation; however, recent lineage-tracing experiments and clonal analyses have

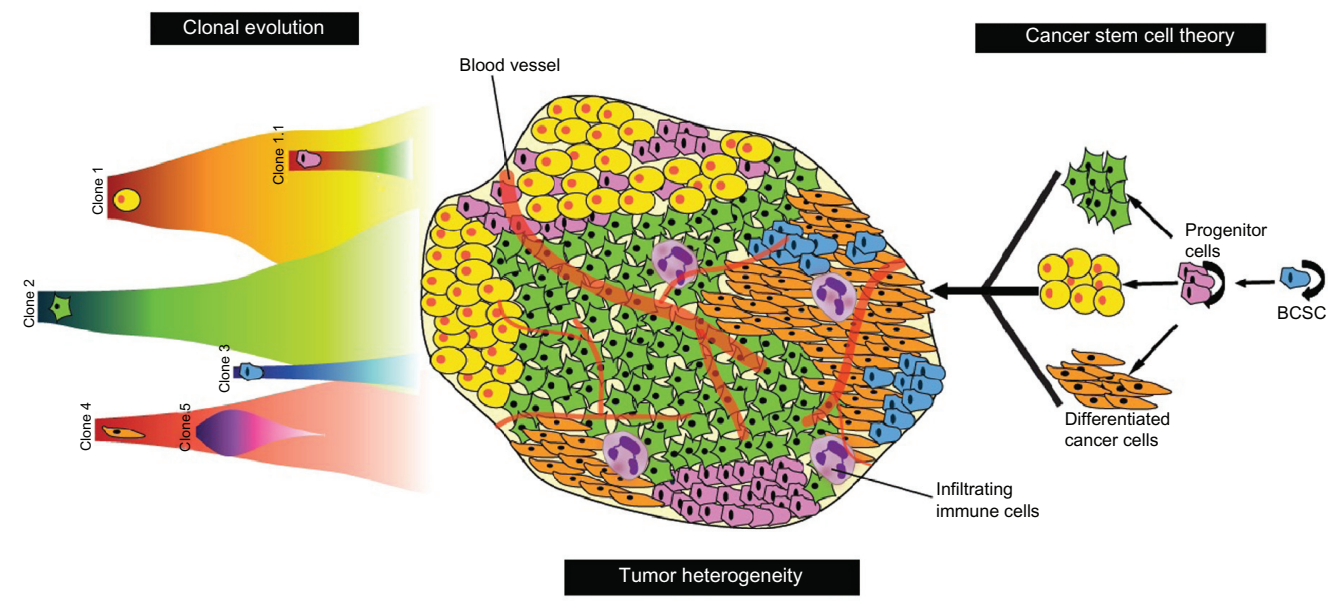

Figure I Models explaining the origins of tumor heterogeneity in breast cancer.

Notes: Tumor heterogeneity (middle) is due to cancer cell types differing in genetic and phenotypic identities, as well as differing in terms of spatial and temporal existence within the tumor. These variations can depend on genetic insults and epigenetic alterations induced by microenvironmental changes. The two models explain the origin of this heterogeneity: the clonal evolution and the BCSC theory. The clonal evolution model (left) proposes that cancer clones compete with each other and the microenvironment to expand and dominate within the tumor (eg, Clone I, 2, 3, and 4). Some clones may expand, but do not evolve fast enough to survive selective pressures and die off (Clone 5). A genetic drift of a single clone can generate a subclone (Clone I.I). The BCSC model (right) proposes that BCSCs arise from transformed mammary cells. These BCSC self-renew (arced arrow), and give rise to highly proliferating progenitor cells that are responsible for generating differentiated cancer cells within the tumor. Abbreviation: BCSC, breast cancer stem cell. 


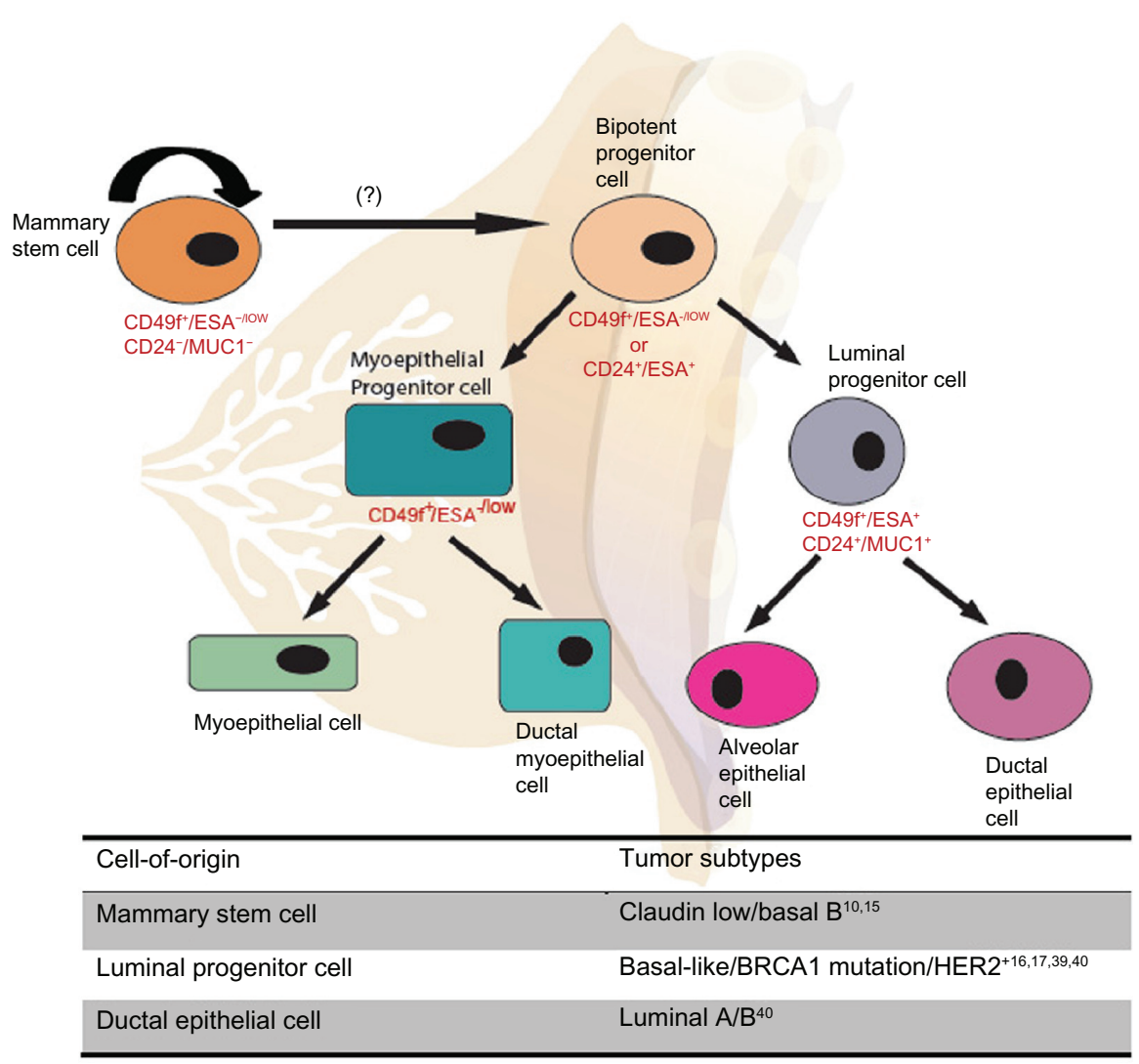

Figure 2 Proposed model of the human MaSC differentiation hierarchy with corresponding surface markers for stem/progenitor cell identification and isolation. Note: A possible relationship to the cellular origin of breast cancer subtypes is shown in Table I.

Abbreviation: MaSC, mammary stem cell.

defined the hierarchical structure of the mouse mammary gland and identified both luminal and myoepithelial longlived unipotent stem/progenitor cells as being able to clonally expand and maintain proliferation in adulthood. ${ }^{24}$ Therefore, these cells could also be a target for cellular transformation. Less is known about the organization of the human mammary gland and most of the data are inferred from experiments that combine flow cytometry, in vitro assays, and xenotransplantation. Human MaSCs have been shown to have a CD49f $\mathrm{f}^{+} /$ $\mathrm{ESA}^{- \text {low }}$ phenotype, suggesting a basal location of these cells in the gland; ${ }^{16,25}$ however, the precise structure of the human MaSCs hierarchy is still not fully understood. For instance, it is unclear whether MaSCs differentiate into a common bipotent progenitor that in turn gives rise to committed progenitors, especially since specific markers for such early lineages are currently lacking. ${ }^{26}$ Indeed, different studies have identified bipotent progenitor cells as luminal $\mathrm{ESA}^{+} / \mathrm{CD} 4 \mathrm{f}^{+} /$ $\mathrm{MUC1}^{-}$or basal $\mathrm{CD} 49 \mathrm{f}^{+} / \mathrm{ESA}^{- \text {/low }}$ cell populations, suggesting that two different stem/progenitor cell populations could also exist in the human breast. ${ }^{26,27}$

BCSCs were first identified and isolated by the virtue of cell-surface expression markers, CD44 and ESA, and the absence of $\mathrm{CD} 24 .^{28}$ Cells identified as $\mathrm{Lin}^{-} / \mathrm{ESA}^{+} / \mathrm{CD}_{4} 4^{+} /$ CD24-/low were found to be more tumorigenic compared to the $\mathrm{CD}_{4} 4^{+} / \mathrm{CD} 24^{+} / \mathrm{ESA}^{-}$cell population, and they were also able to generate tumors in non-obese diabetic/severe combine immunodeficient mice. Importantly, the transplanted tumors recapitulated the same heterogeneity of the original tumor, even after serial transplantation. The molecular characterization of $\mathrm{CD} 44^{+}$and $\mathrm{CD} 24^{+}$cells also confirmed that $\mathrm{CD} 44^{+}$cells express basal stem cell markers, while CD24 cells express markers characteristic of differentiated luminal cells. ${ }^{29}$ However, only a fraction of the $\mathrm{CD} 44^{+} / \mathrm{CD} 24^{- \text {llow }}$ cells are highly tumorigenic, indicating that expression of these markers can be used to enrich BCSCs, but they may not identify a pure CSC population. ${ }^{30,31}$ In response, other breast cancer stem cell markers have been investigated. ALDH1 has been shown to be a BCSC marker, and cancer cells that have a $\mathrm{CD} 44^{+} / \mathrm{CD} 24^{- \text {low }} / \mathrm{ALDH} 11^{\text {high }}$ profile are more tumorigenic, with as few as 20 cells being able to generate tumors after transplantation. ${ }^{32}$ However, ALDH1 activity has been shown to be low or absent in normal mammary stem cells, but high in luminal progenitor cells, again questioning the true identity of BCSCs. ${ }^{33}$ 
BCSCs can also be enriched and cultured in non-adherent conditions through their ability to grow in suspension, bypassing anoikis and forming 'mammospheres'. ${ }^{34}$ The generation of mammospheres can be used to measure the proliferative and the self-renewal abilities of BCSCs at clonal density, with stem and early progenitor cells forming a greater number of mammospheres compared to committed cells over the course of several generations. Importantly, mammosphere cultures enriched with undifferentiated cells demonstrated increased tumor-initiating capacity in vivo. ${ }^{30,35}$ Mammospheres comprise a heterogeneous cell population and it is not known whether clonal sphere-forming cells represent stem/progenitor cells with basal characteristics, luminal characteristics, or both. An elegant study conducted by Pece et al identified normal mammary sphere-forming cells as quiescent MaSCs (which are high retainers of the PKH26 tracking dye), and these were found to be expressing CD24, DLL, DNER, and CD49f. ${ }^{36}$ PKH26 ${ }^{\text {high }}$ stem cells selfrenew asymmetrically giving rise to luminal or myoepithelial progenies. Importantly, cells with this molecular signature were found to be particularly enriched in poorly differentiated breast cancers. ${ }^{36}$ The frequency of BCSCs, characterized by the described markers, depends on the tumor subtype and histological grade, with high-grade tumors being the most enriched. ${ }^{37}$

It is now becoming evident that different breast cancer subtypes may have different cellular origins (Figure 2). Several studies have shown that primitive basal MaSCs are the likely cell of origin of basal B/claudin low and metaplastic cancers, whereas luminal progenitors can generate luminal and basal-like tumors. Indeed, transformation of luminal $\mathrm{ESA}^{+}$progenitor cells by oncogene overexpression can give rise to both $\mathrm{ER}^{+}$(luminal) and $\mathrm{ER}^{-}$(basal-like and possibly HER $2^{+}$) cancers. ${ }^{12,15,16,38}$ Consistent with this notion are recent findings showing that luminal progenitors are the cell of origin for BRCA1 and TP53 mutated basal-like breast cancers. ${ }^{16,17,39}$

\section{Controversies of the BCSC model}

To date, in vivo xenograft and in vitro differentiation data suggest that the human mammary gland is organized in a hierarchical fashion, supporting the BCSC hypothesis. ${ }^{40}$ However, the molecular identity of progenitor cells remains elusive, and it is therefore uncertain whether BCSCs represent transformed MaSCs, progenitor cells, or both. Recent lineage tracing experiments have shown how mouse skin, intestine, and brain CSCs initiate and sustain tumors in their own environment. ${ }^{27,38,41}$ These studies, which elegantly demonstrate
CSC activity in intact tumors, offer much promise for the CSC debate. Similar experiments conducted with BCSCs would greatly benefit our understanding of breast cancer even if the recapitulation of the human disease in mice remains a limitation. ${ }^{42}$ At present, transplantation into humanized mammary fat pads of immunocompromised mice is the best available assay for testing human BCSC function. It is thought that BCSCs are highly tumorigenic, with only a small number of cells required to form tumors when compared to non-stem cells. ${ }^{20}$

This assumption has been a subject of criticism. First, the tumorigenic behavior of cancer cells may vary probabilistically and, given optimal conditions, any tumor cell may have the same probability of exhibiting tumorigenic behavior. ${ }^{43}$ Human tumor cells are not easily conducive to engraftment due to differences in the microenvironment of the mouse mammary fat pad. ${ }^{44} \mathrm{~A}$ second argument refers to the level of immunosuppression in some mouse models. ${ }^{45} \mathrm{~A}$ study by Quintana et al examining melanoma showed that the tumorigenicity of cancer cells can be dramatically increased by transplantation into non-obese diabetic/severe combine immunodeficient gamma mice, which is a mouse strain with superior immunodeficiency. ${ }^{46}$ This study suggests that putative BSCSs may be more tumorigenic simply because of preferential or improved engraftment ability. ${ }^{41}$

Since the initial publication by Al-Hajj et al, which identified $\mathrm{BCSC}$ s as a $\mathrm{Lin}-/ \mathrm{ESA}^{+/} \mathrm{CD} 44^{+} / \mathrm{CD} 24^{- \text {llow }}$ (very similar to the $\mathrm{CD} 49 \mathrm{f}^{+} / \mathrm{ESA}^{-1 / \mathrm{low}} / \mathrm{MUC1}^{-}$phenotype), other studies have followed and they have identified BCSCs as being from the CD44 $/ \mathrm{CD} 24^{- \text {llow }}$ population. ${ }^{28,29,47}$ However, the observation that luminal tumors contain a minimal or non-existent $\mathrm{CD} 44^{+} /$ $\mathrm{CD} 24^{-}$cell population, and the finding that $\mathrm{CD} 44^{-}$cells are also tumorigenic in serial dilution transplantation, questions the true identity of BCSCs and creates doubt in that CD44+ cells may simply represent cells with better engraftment potential. ${ }^{31}$ Indeed, a degree of developmental plasticity has been observed in the BCSC hierarchy, whereby $\mathrm{CD} 44^{+} / \mathrm{CD} 24^{+}$ and $\mathrm{CD} 44^{+} / \mathrm{CD} 24^{-}$cells can interconvert into one another and can generate tumors after xenotransplantation. ${ }^{48}$ Interestingly, the $\mathrm{CD} 44^{+}$cell signature is associated with a high risk of distant metastasis, even if metastatic lesions are enriched with luminal $\mathrm{CD} 24^{+}$cells. ${ }^{29}$ This indicates a phenotypic switch during tumor progression that is independent of the hierarchical differentiation program. This idea is consistent with the clinical observation that $\mathrm{CD} 44^{+} / \mathrm{CD} 24^{-}$cells are not correlated with breast cancer progression or prognosis, but favor distant metastasis. ${ }^{29}$ Therefore, the notion that the $\mathrm{CD} 44^{+} / \mathrm{CD} 24^{-}$phenotype represents a universal BCSCs 
profile is somewhat simplistic, although basal cells identified by this profile seem to be the cell-of-origin of claudin-low/ basal B breast cancer. ${ }^{16}$

Another assumption of the stem cell hierarchy model is that BCSCs are a rare population of cells, but this may not necessarily be the case. Indeed, claudin-low, basal-like, and HER2 ${ }^{+}$cancers are highly enriched for BCSCs, and this characteristic is also retained in cancer cell lines derived from their respective primary tumors. ${ }^{12}$ The abundance of BCSCs is regulated by self-renewal mechanisms which are dependent on the function of the tumor suppressor gene TP53. Mutated or attenuated TP53 signaling confers symmetrical self-renewal, whereby cells can give rise to two identical BCSCs at each round of cell division. Under these conditions, the MaSC differentiation process is compromised and shifted towards an accumulation of undifferentiated BCSCs. Cell tracking experiments have proven symmetrical self-renewal in mammospheres generated from HER2 ${ }^{+}$transgenic mouse tumors and human basal cancer cell lines (personal observation). ${ }^{49}$ Altogether, this evidence casts some doubt on the BCSC hierarchy model, and it may explain why the BCSC hypothesis is not universally accepted by the scientific and medical communities. The key challenge of the BCSC model is the identification of the "primitive malignant stem cell" at the origin of different breast cancers, and recent evidence suggests that this model will need to evolve to accommodate stochastic events that contribute to inter- and intratumor phenotypic and genotypic heterogeneity. As an alternative, tumor heterogeneity can be explained by the clonal evolution model.

\section{An alternative model: clonal evolution}

In contrast to the stem cell hierarchy theory, the clonal evolution model proposes that different clones of cancer cells arise with different selection pressures and microenvironment influences, which can include endogenous and exogenous factors. ${ }^{50}$ Clones of transformed cells can accumulate when cellular hyperproliferation is combined with genetic instability. In this way, tumor heterogeneity is caused by noisedriven gene expression differences, as well as the growth of transformed cells that do not necessarily involve stem cells. ${ }^{51}$ Tumorigenesis is the result of a collection of random mutations that are associated with the appearance of dominant cell clones with growth advantages resulting from activated oncogenes and/or inactivated tumor suppressor genes that are selected by a Darwinian process. ${ }^{52}$ The transformation of cells through genetic mutations is a stochastic process, whereby the phenotypic change in a cell is not predetermined; rather, the different cell clones are generated by random mutation hits. Through Darwinian selection, clones with advantageous mutations are selected to be dominant within the tissue, whereas disadvantageous mutations are discarded. The neutral clones are retained within the population causing a genetic drift. As a consequence, a selection of clones produces a dynamic state during cancer progression: some clones have no desirable mutations for further survival, while others have a selective advantage. Therefore, different parts of the tumor could be undergoing different selective pressure, owing to heterogeneity (Figure 1). ${ }^{53}$

When considering clonal heterogeneity, there is evidence that supports a close clonal relationship between the primary and metastatic tumors. However, in some tumors, metastatic spread occurs at the early stages of tumor evolution, hence the primary and metastatic tumors may evolve to have distinct genetic identities over time. ${ }^{53}$ The first report that investigated clonal diversity of breast tumors at the single cell level was published in $2011 .^{54}$ This study reported the existence of punctuated clonal expansion with few persistent intermediates during tumor progression, rather than a gradual expansion of tumorigenic cells. A similar observation was recently reported in a study of next generation sequencing of 104 primary triple-negative breast cancer cases. ${ }^{55}$ At the time of diagnosis, these tumors displayed a wide spectrum of mutation heterogeneity and clonal evolution. Mutations in the TP53, PIK3CA, and PTEN genes seemed dominant compared to other genetic defects, but they were sometimes present at such low frequencies that they did not appear to be cancer founder mutations. Therefore, mutational heterogeneity is present at the onset of triple negative breast cancer, and patients present with either low-clonality or high-clonality cancers.

Clonal evolution can also explain the phenomenon of intertumor heterogeneity observed in different cancer subtypes. One study that analyzed the contribution of germline and somatic alterations in a cohort of 2000 breast cancers revealed considerable tumor heterogeneity, and highlighted novel subtypes occurring with different frequencies in the population. ${ }^{14}$ However, genetic heterogeneity may not solely explain the phenotypic diversity of tumor cancer cells, as cancer cell behavior can also be influenced by the environment, which can alter gene expression by epigenetic modifications. The microenvironment is not homogenous in a tumor, as different regions within the tissue have varying densities of vasculature, different numbers and types of immune cells, and varying compositions of the extracellular matrix. Again, 
on its own, the clonal evolution model may not fully explain the complexity of tumor heterogeneity as it needs to take in to account non-genetic (epigenetic) influences on hereditable phenotypes.

\section{Stem cell plasticity and cancer: a unifying idea}

It is becoming widely accepted that the BCSC and clonal evolution models are not mutually exclusive; they both contribute to the explanation of tumor heterogeneity. A unifying idea is presented that discusses the role of the inherent developmental plasticity of stem cells. Stochastic events that affect stem cell function - either genetic alterations or epigenetic modifications induced by the tumor microenvironment - can induce cellular transformation and confer cancer cells with stem cell-like characteristics. Although cell-lineage restriction programs are established during embryonic development, adult stem cells maintain a degree of plasticity which is necessary for tissue repair and/or turnover. ${ }^{19}$ This flexibility is maintained by reversible epigenetic modifications which regulate gene expression in a cell-specific manner. Epigenetic modification of the chromatin regulates gene expression without changing the DNA sequence. This is accomplished via DNA methylation, modification of histone tails, and modulation by non-coding RNAs such as microRNA (miRNA). ${ }^{56}$ With changes in chromatin conformation, epigenetic modifications establish heritable transcriptional states responsible for the maintenance of cell identity and function. Epigenetic alterations are observed at the early stages of carcinogenesis, and they play a critical role in tumor initiation and CSC plasticity. Normal stem cells are vulnerable to epigenetic defects when induced to sustained self-renewal, resulting in silencing of tumor suppressor genes. ${ }^{57,58}$

Many tumor suppressor genes are developmentally regulated-genes that regulate the fate of stem cells. Their epigenetic silencing can generate CSCs locked in a selfrenewal state with impaired differentiation potential; indeed, several studies have shown that tumor suppressor genes are more likely to become silenced by DNA methylation in cancer. ${ }^{59-61}$ Reprogramming experiments have demonstrated that epigenetic landscapes are plastic and that they can be influenced and manipulated to change cell fate. The ability of stem cells, progenitor cells, or differentiated cells to transform into CSCs shows the intrinsic plasticity of these cells. ${ }^{19}$ Cells can acquire several rounds of carcinogenic insults before transformation, with progeny being susceptible to further insults, resulting in genetically and sometimes phenotypically different cancer cells. ${ }^{20}$ Since cellular and molecular phenotypes can be determined by genetic and epigenetic alterations affecting differentiation programs, it can be problematic to trace the cell-of-origin of different cancer types. Therefore, it is paramount to stress that the similarities of the genetic signatures between normal MaSCs and BCSCs do not necessarily reflect their direct association during transformation. For instance, BRCA1 and TP53 mutations can affect the differentiation potential of luminal progenitor cells, leading to a basal tumor phenotype. ${ }^{16,17,39}$

Phenotypic switches in response to stochastic events, is one of the characteristics of CSCs. These involve the coexistence of different genetic and epigenetic states during cancer progression. ${ }^{19}$ BCSCs can shift between a stem cell and a non-stem cell state, owing to its plasticity. This was first identified in breast cancer cell lines where non-stem cells $\left(\mathrm{CD} 44^{+} / \mathrm{CD} 24^{+}\right)$were able to generate $\mathrm{CD} 44^{+} / \mathrm{CD} 24^{-} \mathrm{BCSCs}$ with tumorigenic properties and vice versa, depending upon activation of the Activin/Nodal pathway. ${ }^{48}$ One prediction of the stochastic and cell plasticity model is that the proportion of cell populations in a given tumor is determined by a phenotypic equilibrium reached over time. Using Markov's mathematical model, it has been shown that the change in the state of a cancerous cell is not predetermined by its previous cellular memory, but by its ability to maintaining equilibrium to reach a stable state. This is exemplified in breast cancer cell lines whereby BCSCs, luminal, and basal cells isolated according to the expression of a panel of cell surface markers, can change their phenotype over time into a metastable state. This metastable state is characterized by the same phenotype of the parental cell line, with luminal and basal committed cells each giving rise to a similar proportion of BCSCs of the parental cell line. ${ }^{62}$ Therefore, selective pressure could cause an interconversion of these cellular states, so that a metastable cell state is generated and dominates the tumor population. Consistent with this model, BCSCs could arise from more differentiated cells, following a bidirectional interconversion along the stem cell hierarchy. ${ }^{63}$ Based on this notion it is easy to reconcile how selective mutations that confer cancer cells with self-renewal ability can create dominant clones with BCSC characteristics during tumor progression. Different dominant clones could harbor distinct genetic alterations, and these could be selected independently under selective pressure (Figure 3).

Selective pressure can also take control of normal developmental processes and affect CSC plasticity. ${ }^{64}$ Epithelial-to-mesenchymal transition (EMT) a reversible embryonic program that allows for a transition between cellular phenotypes during gastrulation, is recapitulated 
during tumor progression and metastasis when cells change from an epithelial to a motile mesenchymal phenotype. Motile cancer cells can therefore invade neighboring and distant tissues and then colonize new sites after undergoing a reverse mesenchymal-to-epithelial transition (MET). ${ }^{20}$ This interconversion explains the previously described plasticity of $\mathrm{CD} 44^{+} / \mathrm{CD} 24^{-}$cells, and the enrichment for $\mathrm{CD} 24^{+}$cells at the site of metastasis as cells undergo MET. EMT, induced by tumor microenvironment signals (TGF $\beta$, Notch, EGF, Hedgehog, Wnt) induces BCSC properties, such as self-renewal and metastatic ability, in non-tumorigenic cells. ${ }^{65,66}$ TGF $\beta$ and other cytokines produced by the tumor microenvironment are directly involved in the epigenetic regulation of EMT, as well as in the acquisition of the BCSC phenotype. This effect is mediated by DNA methylation alterations that can cause silencing of adhesion molecules (eg, hypermethylation of $C D H 1$ or E-Cadherin) and/or acti- vation of EMT inducers by DNA hypomethylation. ${ }^{66}$ DNA methylation analysis of $\mathrm{CD} 44^{+} / \mathrm{CD} 24^{-}$cells isolated from neoplastic breast tissue shows hypomethylation of several transcription factors involved in EMT, including the transcription factor FOXC1. ${ }^{67} \mathrm{TGF} \beta$ can also induce epigenetic silencing of miR-200a, a key microRNA (miRNA) involved in the regulation of EMT by inducing over-expression of the histone deacetylase SIRT1 and DNA methylation at the gene promoter region.

Two miRNA subfamilies, miR-200c/141 and miR$200 \mathrm{a} / 200 \mathrm{~b} / 429$, are involved in the negative regulation of EMT, as they target EMT-inducing transcription factors ZEB1 and ZEB2. ${ }^{6}$ Both miR-200 gene cluster promoters are frequently hypermethylated, and the epigenetic silencing of miR-200c has been found in BCSCs. ${ }^{69}$ In addition, silencing of miRNAs negatively affects stem cell differentiation and induces self-renewal. This has been observed for miR200c

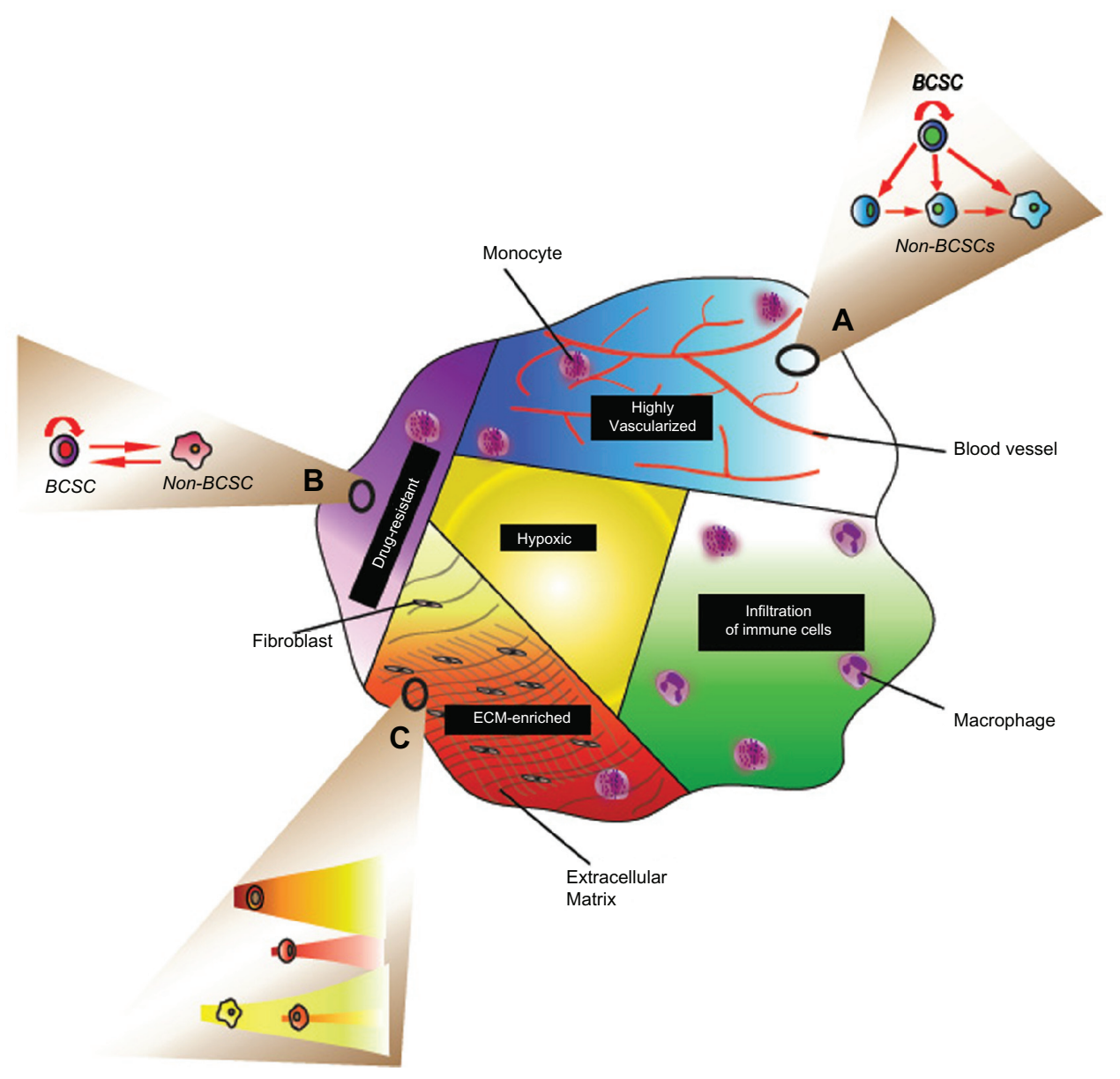

Figure 3 Unified theory of tumor heterogeneity.

Notes: The dynamic nature of tumor initiation and progression is characterized by genetic and epigenetic insults on putative cancer cells and the microenvironment. The heterogeneity of the microenvironment includes varying densities of extracellular matrix, vasculature, immune cells, and oxygen concentration that places selective pressure on cancer cells. The genetic and epigenetic make-up of cells within tumors will vary from one region to the other. Some regions may be characterized by a BCSC hierarchy (A), stochastic plasticity (B), and clonal evolution (C) simultaneously, increasing its complexity.

Abbreviations: BCSC, breast cancer stem cells; ECM, extracellular matrix. 
given that epigenetic silencing is associated with sustained expression of the stem cell self-renewal-regulating factors KLF4 and BMI1 ${ }^{69}$ Another example is the lethal-7 (let-7) gene, whose silencing is directly associated with a BCSC phenotype. ${ }^{70}$ The epigenetic silencing of let-7e mediated by the histone H3K4me3 demethylase JARID1B, over-expressed in breast cancer, contributes to cell cycle progression and the proliferation of cancer cells..$^{71,72}$ Over-expression of other epigenetic modifiers can also induce BCSC plasticity. For instance, high levels of the polycomb protein EZH2 have been found in high grade breast cancers, and it plays a fundamental role in the regulation of stem cell self-renewal and differentiation. ${ }^{73,74}$

The type of epigenetic alterations involved in the generation of BCSCs may depend on their normal cell-of-origin, and therefore different cancer subtypes could acquire clonal characteristics. This is demonstrated by the fact that transformation of genetically identical but phenotypically distinct breast epithelial cells can result in different cancer types. ${ }^{15,75}$

\section{Can we target BCSC plasticity? The ultimate answer to the ultimate question}

Tumor heterogeneity presents a major clinical problem. Important hurdles for successful treatment approaches include treating tumor subtypes with specific therapies and targeting elusive BCSCs. Breast cancer management involves a combination of surgery, chemotherapy, radiotherapy, and some targeted therapies such as hormonal therapy and the use of monoclonal antibodies. ${ }^{2}$ Although chemotherapy and radiotherapy are able to debulk the tumor mass, the majority of patients with basal or HER $2^{+}$cancer subtypes will relapse due to a minimal residual disease (MRD). BCSCs are directly implicated in MRD because of their intrinsic and extrinsic characteristics that are ultimately responsible for tumor recurrence. ${ }^{20} \mathrm{CSC}$ s have an innate chemo- and radio-resistance due to the expression of drug transporters and detoxifying enzymes; altered DNA damage response mechanisms, such as resistance to apoptosis; enhanced DNA repair mechanisms; and quenching of reactive oxygen species. ${ }^{20}$ Stochastic events and adjuvant chemotherapy treatments can act as external factors that can generate resistant BCSCs clones. Such foci of surviving cells are subject to selective pressure and may lead to the development of complex drug resistance mechanisms and increased aggressiveness of the resistant cells (it should be noted that drug resistance can involve endocrine therapies as well). ${ }^{76}$ Hormonal therapy fails to target ER ${ }^{-}$ BCSCs, but they also induce the conversion of cancer cells to BCSCs. ${ }^{77}$ Since the adaptation and selection of different BCSC clones depend on cancer treatments, it is essential that future research efforts employ cancer patient stratification for more personalized treatment approaches.

Many studies are now focusing on targeting BCSC signaling pathways involved in stem cell self-renewal, such as Notch, Hedgehog, and Wnt; these have been extensively reviewed elsewhere. ${ }^{78-80}$ Other strategies have considered targeting EMT, which can be at the core of BCSC plasticity. Even if limited to the targeting of BCSCs with a basal phenotype, this strategy is of considerable interest ${ }^{81}$ however, targeting of EMT on its own may not be a sufficient approach, as a crosstalk between embryonic signaling pathways exists. For instance, the TGF $\beta$ pathway, a main inducer of EMT, is known to interact with Wnt, Notch, and Hedgehog. ${ }^{78}$ There is also evidence that ER signaling influences EMT and the induction of BCSCs. ${ }^{77}$

The tumor microenvironment should also be considered for the development of BCSC-targeted therapies, and integrin-related signaling components, BCSC-surface markers, and stroma-secreted cytokines could be potential targets. ${ }^{82}$ The developmental plasticity of BCSCs as well as the reversible nature of the epigenetic alterations that regulate their function have led to the development of epigenetic therapies as new treatment options. Because epigenetic drugs can restore normal tissue homeostasis, they are a promising tool for differentiation therapy ${ }^{83}$ The idea that BCSCs can be reset to their normal function via modification of their epigenetic landscape is a desired prospect. Tumorigenicity of breast cancer cells harboring genetic defects can be abolished by epigenetic reprogramming, leading to reactivation of silenced tumor suppressor genes. ${ }^{84}$ Therefore, one can envision a therapeutic approach aimed at modulating dominant non-genetic defects to control stem cell function.

Epigenetic drugs that inhibit DNA methylation (DNMT inhibitors) and histone deacetylation (HDAC inhibitors) can restore the expression of silenced tumor suppressor genes, and these drugs are particularly effective for the treatment of leukemia. For instance, the DNA demethylating agent 5-aza-2'-deoxycydine (AZA) has been shown to inhibit self-renewal of leukemic CSCs. ${ }^{85}$ In 2009, Stand Up to Cancer began testing epigenetic drugs on BCSCs and assessed their efficacy for the treatment of HER2 ${ }^{+}$and triple-negative breast cancer in clinical trials. Preclinical studies have shown that two demethylating agents, Decitabine and AZA at low and transient doses, induce the inhibition of BCSC growth as mammospheres due to a decrease in the $\mathrm{CD} 44^{+} / \mathrm{CD} 24^{-} /$ $\mathrm{ALDH}^{+}{ }^{+}$stem cell population across breast cancer cell lines. 
This treatment can also reduce the growth of patient-derived BCSC tumor xenografts due the underlying reactivation of tumor suppressor genes, and also due to the alteration of major cancer cell signaling pathways. ${ }^{86}$ These exciting results suggest that epigenetic therapies can become a reality as they can directly target BCSCs both in vitro and in vivo.

\section{Conclusion}

Breast cancer heterogeneity profoundly impacts the clinical management of the disease. Because of the inter- and intraheterogeneity of breast tumors, classical and targeted therapies are not always successful in eradicating the disease, resulting in poor patient outcome. Cancer recurrence and metastasis are the main cause of poor patient survival, both of which are caused by expansion of MRD. Many studies have shown that tumor heterogeneity arises from BCSCs, whereas others have demonstrated that it is actually the result of clonal evolution. While BCSC and clonal evolution studies show extensive evidence in support of their arguments, caveats in both models still remain. A unified model based on stem cell plasticity can reconcile both views and account for clinical and molecular characteristics of different breast tumor subtypes. According to the stem cell plasticity model, tumor progression is highly dynamic, with cancer cells constantly exposed to internal and external survival pressures. In this context, different areas of the tumor are affected differently by the microenvironment. Therefore, cells may be selected according to clonal evolution, stochastic plasticity, or according to the BCSC differentiation hierarchy at different times and in different regions of the tumor (Figure 3).

Careful assessments of tumor heterogeneity and its microenvironment are therefore needed to devise targeted therapeutic strategies, as generalized interventions can result in the selection of the most resistant cancer cell population and recurrence of a more aggressive form of the disease. Cancer cell plasticity presents a clinical challenge, and future research should focus on understanding how to control its intrinsic and extrinsic effectors; this is certainly not an easy quest and novel avenues should be considered. In this light, epigenetic therapies may offer new solutions. By reversing the epigenetic landscape of cancer cells, epigenetic drugs can reset cancer cell plasticity and cause cancer cells to revert to normalcy. This approach might be instrumental as a new approach in the management of the disease, insofar as cancer could be treated as a chronic condition.

\section{Acknowledgment}

The authors acknowledge the University of Nottingham, the Royal Society of London, and Evocell Ltd for funding.

\section{Disclosure}

The authors report no conflicts of interest in this work.

\section{References}

1. Jemal A, Bray F, Center MM, Ferlay J, Ward E, Forman D. Global cancer statistics. CA Cancer J Clin. 2011;61(2):69-90.

2. Almendro V, Fuster G. Heterogeneity of breast cancer: etiology and clinical relevance. Clin Transl Oncol. 2011;13(11):767-773.

3. Tavassoli FA, Millis RR, Boecker W, Lakhani SR. Lobular Neoplasia. In Tavassoli FA, Devilee P. et al. World Health Organization Classification of Tumours. Lyon, France: IARC Press; 2003:60-62.

4. Reis-Filho JS, Pusztai L. Gene expression profiling in breast cancer: classification, prognostication, and prediction. Lancet. 2011; 378(9805):1812-1823.

5. Huang TH, Esteller M. Chromatin remodeling in mammary gland differentiation and breast tumorigenesis. Cold Spring Harb Perspect Biol. 2010;2(9):a004515.

6. Hanahan D, Weinberg RA. Hallmarks of cancer: the next generation. Cell. 2011;144(5):646-674.

7. Sorlie T, Perou CM, Tibshirani R, et al. Gene expression patterns of breast carcinomas distinguish tumor subclasses with clinical implications. Proc Natl Acad Sci U S A. 2001;98(19):10869-10874.

8. Sorlie T, Tibshirani R, Parker J, et al. Repeated observation of breast tumor subtypes in independent gene expression data sets. Proc Natl Acad Sci U S A. 2003;100(14):8418-8423.

9. Sotiriou C, Neo SY, McShane LM, et al. Breast cancer classification and prognosis based on gene expression profiles from a population-based study. Proc Natl Acad Sci U S A. 2003;100(18):10393-10398.

10. Perou CM, Sorlie T, Eisen MB, et al. Molecular portraits of human breast tumours. Nature. 2000;406(6797):747-752.

11. Calza S, Hall P, Auer G, et al. Intrinsic molecular signature of breast cancer in a population-based cohort of 412 patients. Breast Cancer Res. 2006;8(4):R34.

12. Prat A, Perou CM. Deconstructing the molecular portraits of breast cancer. Mol Oncol. 2011;5(1):5-23.

13. Podo F, Buydens LM, Degani H, et al; for FEMME Consortium. Triplenegative breast cancer: present challenges and new perspectives. Mol Oncol. 2010;4(3):209-229.

14. Curtis C, Shah SP, Chin SF, et al; for METABRIC Group. The genomic and transcriptomic architecture of 2,000 breast tumours reveals novel subgroups. Nature. 2012;486(7403):346-352.

15. Keller PJ, Arendt LM, Skibinski A, et al. Defining the cellular precursors to human breast cancer. Proc Natl Acad Sci U S A. 2012;109(8): 2772-2777.

16. Lim E, Vaillant F, Wu D, et al. Aberrant luminal progenitors as the candidate target population for basal tumor development in BRCA1 mutation carriers. Nat Med. 2009;15(8):907-913.

17. Molyneux G, Geyer FC, Magnay FA, et al. BRCA1 basal-like breast cancers originate from luminal epithelial progenitors and not from basal stem cells. Cell Stem Cell. 2010;7(3):403-417.

18. Allred DC, Brown P, Medina D. The origins of estrogen receptor alphapositive and estrogen receptor alpha-negative human breast cancer. Breast Cancer Res. 2004;6(6):240-245.

19. Shah M, Allegrucci C. Stem cell plasticity in development and cancer: epigenetic origin of cancer stem cells. In: Kundu TK, editor. Epigenetics: Development and Disease. Springer: Subcellular Biochemistry; 2012:61.

20. Visvader JE, Lindeman GJ. Cancer stem cells in solid tumours: accumulating evidence and unresolved questions. Nat Rev Cancer. 2008;8(10):755-768.

21. Badve S, Nakshatri H. Breast-cancer stem cells-beyond semantics. Lancet Oncol. 2012;13(1):e43-e48.

22. Stingl J, Eirew P, Ricketson I, et al. Purification and unique properties of mammary epithelial stem cells. Nature. 2006;439(7079):993-997.

23. Shackleton M, Vaillant F, Simpson KJ, et al. Generation of a functional mammary gland from a single stem cell. Nature. 2006; 439(7072):84-88. 
24. Van Keymeulen A, Rocha AS, Ousset M, et al. Distinct stem cells contribute to mammary gland development and maintenance. Nature. 2011;479(7372):189-193.

25. Eirew P, Stingl J, Raouf A, et al. A method for quantifying normal human mammary epithelial stem cells with in vivo regenerative ability. Nat Med. 2008;14(12):1384-1389.

26. Raouf A, Sun Y, Chatterjee S, Basak P. The biology of human breast epithelial progenitors. Semin Cell Dev Biol. 2012;23(5):606-612.

27. Chen J,LiY,YuTS, etal.A restricted cell population propagates glioblastoma growth after chemotherapy. Nature. 2012;488(7412):522-526.

28. Al-Hajj M, Wicha MS, Benito-Hernandez A, Morrison SJ, Clarke MF. Prospective identification of tumorigenic breast cancer cells. Proc Natl Acad Sci U S A. 2003;100(7):3983-3988.

29. Shipitsin M, Campbell LL, Argani P, et al. Molecular definition of breast tumor heterogeneity. Cancer Cell. Mar 2007;11(3):259-273.

30. Ponti D, Costa A, Zaffaroni N, et al. Isolation and in vitro propagation of tumorigenic breast cancer cells with stem/progenitor cell properties. Cancer Res. 2005;65(13):5506-5511.

31. Fillmore CM, Kuperwasser C. Human breast cancer cell lines contain stem-like cells that self-renew, give rise to phenotypically diverse progeny and survive chemotherapy. Breast Cancer Res. 2008;10(2):R25.

32. Ginestier C, Hur MH, Charafe-Jauffret E, et al. ALDH1 is a marker of normal and malignant human mammary stem cells and a predictor of poor clinical outcome. Cell Stem Cell. 2007;1(5):555-567.

33. Eirew P, Kannan N, Knapp DJ, et al. Aldehyde dehydrogenase activity is a biomarker of primitive normal human mammary luminal cells. Stem Cells. 2012;30(2):344-348.

34. Dontu G, Abdallah WM, Foley JM, et al. In vitro propagation and transcriptional profiling of human mammary stem/progenitor cells. Genes Dev. 2003;17(10):1253-1270.

35. Harrison H, Farnie G, Brennan KR, Clarke RB. Breast cancer stem cells: something out of notching? Cancer Res. 2010;70(22):8973-8976.

36. Pece S, Tosoni D, Confalonieri S, et al. Biological and molecular heterogeneity of breast cancers correlates with their cancer stem cell content. Cell. 2010;140(1):62-73.

37. Charafe-Jauffret E, Ginestier C, Birnbaum D. Breast cancer stem cells: tools and models to rely on. BMC Cancer. 2009;9:202.

38. Driessens G, Beck B, Caauwe A, Simons BD, Blanpain C. Defining the mode of tumour growth by clonal analysis. Nature. 2012;488(7412) 527-530.

39. Proia TA, Keller PJ, Gupta PB, et al. Genetic predisposition directs breast cancer phenotype by dictating progenitor cell fate. Cell Stem Cell. 2011;8(2):149-163.

40. Visvader JE. Cells of origin in cancer. Nature. 2011;469(7330): 314-322.

41. Schepers AG, Snippert HJ, Stange DE, et al. Lineage tracing reveals Lgr5+ stem cell activity in mouse intestinal adenomas. Science. 2012;337(6095):730-735.

42. Gilbertson RJ, Graham TA. Cancer: Resolving the stem-cell debate. Nature. 2012;488(7412):462-463.

43. Kern SE, Shibata D. The fuzzy math of solid tumor stem cells: a perspective. Cancer Res. 2007;67(19):8985-8988.

44. Ronnov-Jessen L, Petersen OW, Bissell MJ. Cellular changes involved in conversion of normal to malignant breast: importance of the stromal reaction. Physiol Rev. 1996;76(1):69-125.

45. Lindeman GJ, Visvader JE. Insights into the cell of origin in breast cancer and breast cancer stem cells. Asia Pac J Clin Oncol. 2010;6(2):89-97.

46. Quintana E, Shackleton M, Sabel MS, Fullen DR, Johnson TM, Morrison SJ. Efficient tumour formation by single human melanoma cells. Nature. 2008;456(7222):593-598.

47. Stingl J. Detection and analysis of mammary gland stem cells. J Pathol. 2009;217(2):229-241.

48. Meyer MJ, Fleming JM, Ali MA, Pesesky MW, Ginsburg E, Vonderhaar BK. Dynamic regulation of CD24 and the invasive, CD44posCD24neg phenotype in breast cancer cell lines. Breast Cancer Res. 2009;11(6):R82.
49. Cicalese A, Bonizzi G, Pasi CE, et al. The tumor suppressor p53 regulates polarity of self-renewing divisions in mammary stem cells. Cell. 2009;138(6):1083-1095.

50. Nowell PC. The clonal evolution of tumor cell populations. Science. 1976;194(4260):23-28.

51. Spencer SL, Gaudet S, Albeck JG, Burke JM, Sorger PK. Non-genetic origins of cell-to-cell variability in TRAIL-induced apoptosis. Nature. 2009;459(7245):428-432.

52. Marusyk A, Almendro V, Polyak K. Intra-tumour heterogeneity: a looking glass for cancer? Nat Rev Cancer. 2012;12(5):323-334.

53. Marusyk A, Polyak K. Tumor heterogeneity: causes and consequences. Biochim Biophys Acta. 2010;1805(1):105-117.

54. Navin N, Kendall J, Troge J, et al. Tumour evolution inferred by singlecell sequencing. Nature. 2011;472(7341):90-94.

55. Shah SP, Roth A, Goya R, et al. The clonal and mutational evolution spectrum of primary triple-negative breast cancers. Nature. 2012; 486(7403):395-399.

56. Shah M, Allegrucci C. Stem cell plasticity in development and cancer: epigenetic origin of cancer stem cells. In: Kundu TK, editor. Epigenetics: Development and Disease. Vol 61. New York: Springer; 2012.

57. Allegrucci $\mathrm{C}, \mathrm{Wu} \mathrm{YZ}$, Thurston $\mathrm{A}$, et al. Restriction landmark genome scanning identifies culture-induced DNA methylation instability in the human embryonic stem cell epigenome. Hum Mol Genet. 2007;16(10):1253-1268.

58. Shen Y, Chow J, Wang Z, Fan G. Abnormal CpG island methylation occurs during in vitro differentiation of human embryonic stem cells. Hum Mol Genet. 2006;15(17):2623-2635.

59. Widschwendter M, Fiegl H, Egle D, et al. Epigenetic stem cell signature in cancer. Nat Genet. 2007;39(2):157-158.

60. Ohm JE, McGarvey KM, Yu X, et al. A stem cell-like chromatin pattern may predispose tumor suppressor genes to DNA hypermethylation and heritable silencing. Nat Genet. 2007;39(2):237-242.

61. Schlesinger Y, Straussman R, Keshet I, et al. Polycomb-mediated methylation on Lys27 of histone $\mathrm{H} 3$ pre-marks genes for de novo methylation in cancer. Nat Genet. 2007;39(2):232-236.

62. Gupta PB, Fillmore CM, Jiang G, et al. Stochastic state transitions give rise to phenotypic equilibrium in populations of cancer cells. Cell. 2011;146(4):633-644.

63. Chaffer CL, Brueckmann I, Scheel C, et al. Normal and neoplastic nonstem cells can spontaneously convert to a stem-like state. Proc Natl Acad Sci U S A. 2011;108(19):7950-7955.

64. Ben-Porath I, Thomson MW, Carey VJ, et al. An embryonic stem cell-like gene expression signature in poorly differentiated aggressive human tumors. Nat Genet. 2008;40(5):499-507.

65. Mani SA, Guo W, Liao MJ, et al. The epithelial-mesenchymal transition generates cells with properties of stem cells. Cell. 2008;133(4): 704-715.

66. Polyak K, Weinberg RA. Transitions between epithelial and mesenchymal states: acquisition of malignant and stem cell traits. Nat Rev Cancer. 2009;9(4):265-273.

67. Bloushtain-Qimron N, Yao J, Snyder EL, et al. Cell type-specific DNA methylation patterns in the human breast. Proc Natl Acad Sci US A. 2008;105(37):14076-14081.

68. Hermeking H. MicroRNAs in the p53 network: micromanagement of tumour suppression. Nat Rev Cancer. 2012;12(9):613-626.

69. Shimono Y, Zabala M, Cho RW, et al. Downregulation of miRNA200c links breast cancer stem cells with normal stem cells. Cell. 2009;138(3):592-603.

70. Zimmerman AL, Wu S. MicroRNAs, cancer and cancer stem cells. Cancer Lett. 2011;300(1):10-19.

71. Mitra D, Das PM, Huynh FC, Jones FE. Jumonji/ARID1 B (JARID1B) protein promotes breast tumor cell cycle progression through epigenetic repression of microRNA let-7e. J Biol Chem. 2011;286(47):40531-40535.

72. Barrett A, Santangelo S, Tan K, et al. Breast cancer associated transcriptional repressor PLU-1/JARID1B interacts directly with histone deacetylases. Int J Cancer. 2007;121(2):265-275.

73. Bracken AP, Dietrich N, Pasini D, Hansen KH, Helin K. Genome-wide mapping of Polycomb target genes unravels their roles in cell fate transitions. Genes Dev. 2006;20(9):1123-1136. 
74. Holm K, Grabau D, Lovgren K, et al. Global H3K27 trimethylation and EZH2 abundance in breast tumor subtypes. Mol Oncol. 2012;6(5):494-506.

75. Ince TA, Richardson AL, Bell GW, et al. Transformation of different human breast epithelial cell types leads to distinct tumor phenotypes. Cancer Cell. 2007;12(2):160-170.

76. Meads MB, Gatenby RA, Dalton WS. Environment-mediated drug resistance: a major contributor to minimal residual disease. Nat Rev Cancer. 2009;9(9):665-674.

77. Smalley M, Piggott L, Clarkson R. Breast cancer stem cells: Obstacles to therapy. Cancer Lett. April 30, 2012. [Epub ahead of print.]

78. Takebe N, Harris PJ, Warren RQ, Ivy SP. Targeting cancer stem cells by inhibiting Wnt, Notch, and Hedgehog pathways. Nat Rev Clin Oncol. 2011;8(2):97-106.

79. Zhou BB, Zhang H, Damelin M, Geles KG, Grindley JC, Dirks PB. Tumour-initiating cells: challenges and opportunities for anticancer drug discovery. Nat Rev Drug Discov. 2009;8(10):806-823.

80. Alison MR, Lim SM, Nicholson LJ. Cancer stem cells: problems for therapy? J Pathol. 2011;223(2):147-161.
81. Kim J, Villadsen R, Sorlie T, et al. Tumor initiating but differentiated luminal-like breast cancer cells are highly invasive in the absence of basal-like activity. Proc Natl Acad Sci U S A. 2012;109(16):6124-6129.

82. Ablett MP, Singh JK, Clarke RB. Stem cells in breast tumours: Are they ready for the clinic? Eur J Cancer. 2012;48(14):2104-2116.

83. Berdasco M, Esteller M. Aberrant epigenetic landscape in cancer: how cellular identity goes awry. Dev Cell. 2010;19(5):698-711.

84. Allegrucci C, Rushton MD, Dixon JE, et al. Epigenetic reprogramming of breast cancer cells with oocyte extracts. Mol Cancer. 2011; 10(1):7.

85. Hu Z, Negrotto S, Gu X, et al. Decitabine maintains hematopoietic precursor self-renewal by preventing repression of stem cell genes by a differentiation-inducing stimulus. Mol Cancer Ther. 2010;9(6): 1536-1543.

86. Tsai HC, Li H, Van Neste L, et al. Transient low doses of DNAdemethylating agents exert durable antitumor effects on hematological and epithelial tumor cells. Cancer Cell. 2012;21(3):430-446.

\section{Publish your work in this journal}

Breast Cancer: Targets and Therapy is an international, peerreviewed open access journal focusing on breast cancer research, identification of therapeutic targets and the optimal use of preventative and integrated treatment interventions to achieve improved outcomes, enhanced survival and quality of life for the cancer patient.
View the full aims and scopes of this journal here. The manuscript management system is completely online and includes a very quick and fair peer-review system, which is all easy to use. Visit http:// www.dovepress.com/testimonials.php to read real quotes from published authors. 